\title{
Approaches to Teaching Proust's Fiction and Criticism edited by Elyane Dezon-Jones and Inge Crosman Wimmers
}

\section{Geneviève Henrot Sostero}

\section{(2) OpenEdition}

Journals

\section{Edizione digitale}

URL: http://journals.openedition.org/studifrancesi/36627

DOI: $10.4000 /$ studifrancesi.36627

ISSN: 2421-5856

\section{Editore}

Rosenberg \& Sellier

\section{Edizione cartacea}

Data di pubblicazione: 1 juillet 2005

Paginazione: 196-197

ISSN: 0039-2944

\section{Notizia bibliografica digitale}

Geneviève Henrot Sostero, «Approaches to Teaching Proust's Fiction and Criticism edited by Elyane Dezon-Jones and Inge Crosman Wimmers», Studi Francesi [Online], 145 (XLIX | I) | 2005, online dal 30 novembre 2015, consultato il 18 avril 2021. URL: http://journals.openedition.org/studifrancesi/36627 ; DOI: https://doi.org/10.4000/studifrancesi.36627

Questo documento è stato generato automaticamente il 18 avril 2021.

\section{cc) (†) $\odot$}

Studi Francesi è distribuita con Licenza Creative Commons Attribuzione - Non commerciale - Non opere derivate 4.0 Internazionale. 


\title{
Approaches to Teaching Proust's Fiction and Criticism edited by Elyane Dezon-Jones and Inge Crosman Wimmers
}

\author{
Geneviève Henrot Sostero
}

\section{NOTIZIA}

Approaches to Teaching Proust's Fiction and Criticism edited by Elyane DEZON-JONES and Inge CROSMAN WIMMERS, New York, The Moderne Language Association of America, 2003, pp.

184.

1 I saggi raccolti in questo volume, inserito in una collana destinata a studenti universitari e ad insegnanti di letteratura dei licei e degli atenei americani, fanno vedere quanto la Recherche si discosti dagli stretti principi critici espressi altrove da Proust, per esplorare gli aspetti estetici, sociali, filosofici, sessuali del suo tempo in modi inediti e sconvolgenti.

2 La prima sezione, "Materials", aggiorna, demistificandola, la complessa storia della pubblicazione del romanzo proustiano. Sono passate in rassegna tutte le fonti importanti oggi conosciute, non solo biografie classiche e recenti, traduzioni autorevoli, saggi e monografie di referenza, ma anche materiali audiovisivi e risorse elettroniche (Materials, Elyane DEZON-JONES e Inge CROSMAN WIMMERS, pp. 3-13). Nel reso conto delle risorse in linea di materiali didattici legati a Proust e alla sua opera, vengono messi in primo piano l'ITEM (Proust at the Institut des Textes et des Manuscrits Modernes, Bernard BRUN, pp. 13-14), la Bibliothèque Nationale de France (Genetics Approches to Proust's Manuscripts, Nathalie MAURIAC DYER, pp. 14-16) e il Kolb-Proust Archive for Research, dell'Università dell'Illinois/Champaing (USA) (Virginie GREEN e Caroline SZYLOWICZ, pp. 17-18). 
3 La seconda sezione, "Approaches", riflette la varietà di riflessioni stimolate oggi dall'opera in un contesto di insegnamento di alto livello. Il primo gruppo di saggi illustra il contesto storico dell'ambiente artistico in cui Proust operava (Proust: A Man of His Times, William C. CARTER, pp. 26-32), il ruolo dell"'Affaire Dreyfus" (The Role of the Dreyfus Affair in the 'Recherche', Joseph BRAMI, pp. 33-37) e del Primo Conflitto Mondiale nella finzione romanzesca (Cataclysm at One Remove: The War in 'Le Temps retrouvé, Edwards J. HUGHES, pp. 38-43). I saggi successivi interpretano la funzione dell'arte e dello sguardo (Proust Art Nouveau?, Françoise LERICHE, pp. 44-49, Proust and Painters, Kasuyoshi YoshIKAWA, pp. 50-55; Seeing Proust seeing, Mary Ann CAWS, pp. 56-60; "Maintenant regardez": Proust in a Postmodem Contexte, Margaret E. GRAY, pp. 61- 65). Il terzo gruppo, "Interpretive Perspectives", analizza la funzione dei nomi, della memoria, della lettura, dell'omosessualità nella Recherche (rispettivamente coding and Decoding: Names in the 'Recherche', Eugene NICOLE, pp. 66-71; A Lesson to Reading: From 'Contre Sainte-Beuve' to the 'Recherche' Pascal A. IFRI, pp. 72-76; In Search of Hidden Impressions, Geneviève HEMOT, pp. 77-87; Proust's Esther, Emily EELLS, pp. 88-94; Intertextual Pedagogy: Proust and Flaubert, Mireille NATUREL, pp. 95-101; Homosexuality in the 'Recherche', Lawrence R. SCHEHR, pp. 102-107). Vengono anche presentate e discusse varie interpretazioni cinematografiche dell'opera (Proust and the Cinema, Rebecca GRAVES, p. 108-112). Gli ultimi interventi sono volti a di fornire agli insegnanti spunti e metodi concreti per insegnare Proust in diversi contesti d'insegnamento letterario e a diversi livelli: l"'explication de texte" di Roger sнAттUск (pp. 113-118), i “morceaux choisis" di Michèle MAGILL (pp. 119-122), il tema dell'architettura di J. Theodore JoHNSON jr. (Reconstructing the Churches of 'Combray', pp. 130-134), la segmentazione del testo (The Segmentation of 'Combray', Julie solomon, pp. 123-129) e la struttura dell'esperienza (Structuring the Proustian Experience, Christie MCDONALD, pp. 135-139), i "Gender Studies" (What to Do with Montjouvain?, Brigitte MAHUZIER (pp. 140-143), il comparatismo di Diane R. LEONARD (Proust, Ruskin and the Visual Arts, pp. 144-150), i rapporti intersemiotici letteratura-musica di Jean-Louis PAUTROT (pp. 151-155). Chiudono il volume delle note biografiche sui collaboratori dei due Mondi, una bibliografia dei lavori citati e un indice dei nomi. Lo spirito pragmatico voluto dalla collana "Approaches to Teaching World Literature" consente di avvicinare studenti e insegnanti a un'opera nota per la sua ricchezza e la sua complessità. 